HIFAN 1317 - LBNL 54917

\title{
A ray-based algorithm for multi-dimensional linear conversion
}

\author{
Eugene R. Tracy \\ Department of Physics, College of William and Mary, \\ Williamsburg, Virginia 23187-8795 \\ ertrac@wm.edu \\ Allan N. Kaufman \\ Lawrence Berkeley National Laboratory \\ and \\ Physics Department, UC Berkeley \\ Berkeley, CA 94720 \\ ankaufman@lbl.gov \\ André Jaun \\ Alfuén Laboratory \\ Royal Institute of Technology \\ SE-100 44 Stockholm \\ jaun@fusion.kth.se
}

(September 5, 2001)

\begin{abstract}
A numerical algorithm is proposed for connecting the incoming and outgoing wave fields in studies of linear conversion. This is the first such ray-based algorithm for wave conversion in multiple spatial dimensions. It is demonstrated that, aside from the overall phase of the coupling, one can directly evaluate all quantities needed for the connection coefficients from the ray geometry. The ray dynamics is generated using the determinant of the dispersion matrix as
\end{abstract}


the hamiltonian. Using information available while following an incoming ray, the algorithm automatically detects that the ray has entered a conversion region, evaluates the transmission and conversion coefficients, and launches the transmitted ray. The algorithm does not require any prior knowledge of the geometry of the conversion region. The algorithm is illustrated using a twodimensional toroidal model with resonant conversion from a magnetosonic to an ion-hybrid wave.

Consider a linear multi-component wave equation in multiple spatial dimensions. An important tool for its analysis is ray tracing (also known as the WKB method), which provides physical insight often difficult to extract from full-wave simulations. For example, ray tracing methods (as developed by Maslov and others [1-4]) reveal how energy propagates through the system, and how fine-scale spatial structure can form due to caustics. For an $n$-dimensional system, the ray dynamics is hamiltonian in the $2 n$-dimensional ray phase space $(\mathrm{x}, \mathrm{k})$.

When the background medium is non-uniform, it often occurs that the dispersion relations for two distinct modes can be simultaneously satisfied in certain localized regions. In such linear conversion regions the WKB approximation breaks down and a full-wave description which connects the incoming and outgoing waves must be developed. Global wavefields have recently been calculated for two-dimensional problems of interest in tokamak heating, including mode-conversion to ion-hybrid and ion-Bernstein waves [5-8]. Such large-scale computations, performed with a limited spatial resolution, do not yet allow for a systematic study of the parameter space. Full-wave numerical calculations are also unlikely to treat problems involving conversion to the electron-Bernstein wave in the near future.

Separating the propagation (which can be treated using WKB) from the conversion, in [4], we showed how to compute the connection formulas, once a reduction to a local $2 \times 2$ coupled wave equation was carried out. Not discussed there, however, was the manner of reduction without prior knowledge of the geometry of the conversion region. This lack of 
a reduction algorithm limited the application of our method to problems simple enough to allow an analytical reduction [9]. In more general situations, the physics will likely be too complex to allow such an intuitive reduction, and one must provide a means for the ray tracing algorithm to 'discover' the conversion region and evaluate the connection formulas automatically.

The long-term goal of this research program is to develop a ray-based algorithm for computing wave fields in complex geometries including linear conversion. Such an algorithm would supplement direct full-wave calculations and provide further insight.

In closed systems, such as a toroidal cavity, conversion can occur repeatedly and has a dramatic effect upon the global structure of the wave fields. The presence of linear conversion also strongly affects the response to external driving (such as antennas, see [10] for a one-dimensional study). A complete numerical algorithm would have the ability to: 1] Launch a family of rays. 2] Propagate both amplitude and phase 'freely' until linear conversion is encountered. 3] Detect that the rays are entering a linear conversion region. 4] Connect the incoming and outgoing wave fields (amplitude and phase) for both the transmitted and converted waves. 5] Construct the full wave fields in the vicinity of the conversion. 6] Reflect waves off boundaries when they are encountered. And, 7] iterate this process as many times as needed to understand where the energy is eventually deposited.

In this paper we describe an algorithm to carry out steps 3] and 4] of this program. It is demonstrated that, except for the phase of the normalized coupling constant $\eta$, the connection formulas and the the transmitted ray can be calculated using information which can be measured while following the incident ray. (The phase of the coupling constant requires more effort and will be discussed in a later paper.) The present conversion algorithm is then demonstrated on a problem of interest in plasma heating: the conversion of a magnetosonic wave (MSW) to the ion-hybrid wave (IHW).

The paper is arranged as follows: we begin by briefly reminding the reader of the phase space formulation of ray tracing. We then describe the algorithm, and end with a summary of its application to the MSW-IHW conversion. 
For concreteness, we consider the propagation of electromagnetic waves in a twodimensional magnetized plasma, representing the poloidal plane of an axisymmetric torus. The algorithm described below can be easily generalized for wave equations in three spatial dimensions, or for problems which include more field variables (e.g. fluid velocity fields, densities, etc.). Assuming the background plasma properties are given and independent of time, the problem reduces to solving for the electric field $\mathbf{E}(\mathbf{x}) e^{-i \omega t}$. Dissipative effects do not affect the ray trajectories and can be treated perturbatively to extract the power absorption profile. Hence, we use the hermitian part of the full $3 \times 3$ dispersion matrix, D. In the $\mathrm{x}$-representation, the electric field satisfies:

$$
\mathrm{D}(\mathrm{x}, \mathrm{k}=-i \nabla ; \omega) \cdot \mathrm{E}(\mathrm{x})=0 .
$$

In what follows, we suppress the $\omega$ dependence. In symbolic form [4], we write the dispersion matrix as the $3 \times 3$ hermitian matrix $D(x, k)$. For any point in the four-dimensional ray phase space, $(\mathrm{x}, \mathrm{k})=\left(x, y, k_{x}, k_{y}\right)$, the matrix $\mathrm{D}(\mathrm{x}, \mathrm{k})$ has three (real) eigenvalues and three associated eigenvectors:

$$
\mathrm{D}(\mathrm{x}, \mathrm{k}) \cdot \hat{\mathrm{e}}_{j}(\mathrm{x}, \mathrm{k})=D_{j}(\mathrm{x}, \mathrm{k}) \hat{\mathrm{e}}_{j}(\mathrm{x}, \mathrm{k}) . \quad j=a, b, c .
$$

In order to simplify the algebraic expressions to come, we now shift notation from $(x, k)$ to $\mathrm{z}=(\mathrm{x}, \mathrm{k})$ for our phase space coordinates.

The dispersion surface of mode $j$ consists of those values of $\mathrm{z}$ such that $D_{j}(\mathrm{z})=0$. In the four-dimensional phase space the dispersion surfaces are three-dimensional. Linear conversion can occur in regions where two of these dispersion functions are nearly zero [11].

First, start with a ray of mode $a$ and launch it far from a conversion region. Ray $a$ is propagated using the full determinant $D(\mathbf{z}) \equiv \operatorname{det}(\mathrm{D})(\mathbf{z})$ as its hamiltonian. That is, $\dot{\mathbf{z}} \equiv d \mathbf{z} / d \sigma_{D}$ is given by:

$$
\frac{d \mathbf{x}}{d \sigma_{D}}=-\frac{\partial D}{\partial \mathbf{k}}, \quad \frac{d \mathbf{k}}{d \sigma_{D}}=\frac{\partial D}{\partial \mathbf{x}} .
$$

The physical time, $t$, is related to the ray parameter $\sigma_{D}$ via $d t / d \sigma_{D}=\partial D / \partial \omega[4]$. Note that in [4] we used $D_{a}$ as the ray hamiltonian. This leads to a different parameterization 
of the ray, but otherwise leaves it unchanged. For notational clarity, we now drop the ' $D$ ' subscript and denote the ray parameter as $\sigma$. We now turn to the question of how to detect that the ray is entering a linear conversion region.

The first task at hand for implementing a numerical ray tracing algorithm which includes mode conversion is to ask: What quantities can be monitored following the rays which detect that one has entered a conversion region? The problem is made more difficult when we add the requirement that the algorithm should provide results that are invariant under changes of representation (i.e. under congruence transformations of the vector wave equation), and under linear canonical transformations. The algorithm proposed here satisfies both of these requirements.

Any quantity which is proposed as a monitor for linear conversion must have the property that it can detect when a second eigenvalue of $D$ is getting small. For a $2 \times 2$ problem, the natural quantity to monitor is $\operatorname{Tr} \mathrm{D}=D_{11}+D_{22}=D_{a}+D_{b}\left(=D_{b}\right.$ since $\left.D_{a}=0\right)$. Following a ray of mode $a$, a local minimum of $|\operatorname{Tr} \mathrm{D}|(\sigma)$ strongly suggests that the ray may have entered a conversion region. For the $3 \times 3$ problem, the trace is not useful as a monitor for conversion beause in this case $\operatorname{Tr} \mathrm{D}=D_{11}+D_{22}+D_{33}=D_{b}+D_{c}$. Congruence transformations leave the signs of the eigenvalues $D_{b}$ and $D_{c}$ invariant, but not their magnitudes, hence the sign of the trace can change under a congruence transformation. A more useful quantity to monitor for the $3 \times 3$ problem is the symmetric function of pairwise products of the eigenvalues, which we denote as $F=D_{a} D_{b}+D_{a} D_{c}+D_{b} D_{c}$ [3]. The function $F$ can be evaluated by expanding $\mathrm{D}$ in minors:

$$
F=\left(D_{22} D_{33}-\left|D_{23}\right|^{2}\right)+\left(D_{11} D_{33}-\left|D_{13}\right|^{2}\right)+\left(D_{11} D_{22}-\left|D_{12}\right|^{2}\right)
$$

On the dispersion manifold $D_{a}=0$ and we have $F=D_{b} D_{c}$. The value of the ray parameter where the relevant monitor, $|\operatorname{Tr} \mathrm{D}|(\sigma)$ or $|F|(\sigma)$, has a local minimum is defined as the point $\sigma_{0}$. It is an important aspect of our algorithm that we can define a single point on the ray as the location where conversion occurs. For a family of rays, it is assumed that we can treat the conversion as occurring ray by ray for the entire family. We now proceed to look 
for further evidence that one is near a conversion.

When the conversion monitor suggests that the ray has entered a conversion region, at the point $\mathbf{z}_{0} \equiv \mathbf{z}\left(\sigma_{0}\right)$ we evaluate the ray velocity and acceleration:

$$
\dot{\mathbf{z}}_{0} \equiv \frac{d \mathbf{z}}{d \sigma}\left(\sigma_{0}\right), \quad \ddot{\mathbf{z}}_{0} \equiv \frac{d^{2} \mathbf{z}}{d \sigma^{2}}\left(\sigma_{0}\right)
$$

Generically, these two vectors are not parallel. Hence, using $\mathbf{z}\left(\sigma_{0}\right) \equiv \mathbf{z}_{0}$ as an origin, they can be used to define a two-dimensional plane embedded in the four-dimensional phase space (see Fig. (1)). This is the osculating plane of the ray [12]. Our computation of the connection coefficients in [4] implicitly assumes that the ray is locally confined to such a plane. Confinement to this two-dimensional plane allows us to reduce the local wave equation in the vicinity of the conversion to a first order ordinary differential equation which is easy to solve. However, if the ray dynamics transverse to the plane is important (i.e. if the ray exhibits the phase space equivalent of torsion), then this reduction is not valid and one must use a higher order description.

Now compute the symplectic 'area' spanned by $\dot{z}_{0}$ and $\ddot{z}_{0}$ :

$$
A \equiv\left|\dot{\mathrm{x}}_{0} \cdot \ddot{\mathrm{k}}_{0}-\dot{\mathrm{k}}_{0} \cdot \ddot{\mathrm{x}}_{0}\right|
$$

and introduce the basis vectors

$$
\hat{\mathrm{e}}_{q} \equiv A^{-1 / 2} \dot{\mathrm{z}}_{0} \quad \hat{\mathrm{e}}_{p} \equiv A^{-1 / 2} \ddot{\mathbf{z}}_{0}
$$

Any point in the osculating plane can be represented as a linear combination of these two vectors:

$$
\mathrm{Z}(q, p)=\mathrm{z}_{0}+q \hat{\mathrm{e}}_{q}+p \hat{\mathrm{e}}_{p}
$$

In what follows, we denote points in the osculating plane by capitals, $\mathrm{Z}$, and we note that $q$ and $p$ are a conjugate pair of coordinates on this plane.

Now consider the restriction of the ray hamiltonian $D(\mathbf{z})$ to the osculating plane, denoted as $h(q, p)$ : 


$$
h(q, p) \equiv D(\mathbf{Z}(q, p))
$$

We note that $h(q, p)$ acts as a local hamiltonian governing the ray dynamics in the neighborhood of the conversion. For conversion to occur, the hamiltonian must have a saddle structure. This ensures that there is a second dispersion manifold in the vicinity, and that the local geometry looks like that of an 'avoided crossing' or a 'tunneling' region (see Fig. (2)). We first assume there is a saddle structure, and ask about the nature of the rays in the vicinity. From this, we uncover an important relationship between the saddle geometry and the coupling coefficient $\eta$. After establishing this connection, we then ask how to find the saddle geometry approximately, using information evaluated only along the incoming ray.

Now consider the directional derivatives (see Eqs. (7)-(9)):

$$
\frac{\partial h}{\partial q}=\hat{\mathrm{e}}_{q} \cdot \nabla_{z} D, \quad \frac{\partial h}{\partial p}=\hat{\mathrm{e}}_{p} \cdot \nabla_{z} D
$$

A saddle point $\left(q_{*}, p_{*}\right)$ satisfies two conditions: 1] the gradient of $h$ vanishes:

$$
\frac{\partial h}{\partial q}\left(q_{*}, p_{*}\right)=\frac{\partial h}{\partial p}\left(q_{*}, p_{*}\right)=0
$$

and 2] the matrix of second derivatives (the hessian)

$$
\mathcal{H}_{*} \equiv\left(\begin{array}{cc}
\frac{\partial^{2} h}{\partial q^{2}} & \frac{\partial^{2} h}{\partial q \partial p} \\
\frac{\partial^{2} h}{\partial q \partial p} & \frac{\partial^{2} h}{\partial p^{2}}
\end{array}\right)_{\left(q_{*}, p_{*}\right)}
$$

at the saddle point must have two non-zero eigenvalues with opposite signs (the determinant of the hessian must be negative). We note that the saddle point $\left(q_{*}, p_{*}\right)$ corresponds to the point $\mathbf{Z}_{*} \equiv \mathbf{Z}\left(q_{*}, p_{*}\right)=\mathrm{z}_{0}+q_{*} \hat{\mathrm{e}}_{q}+p_{*} \hat{\mathrm{e}}_{p}$ in the full phase space. Now expand $h(q, p)$ about the saddle point. To quadratic order, this gives:

$$
h(q, p)=h\left(q_{*}, p_{*}\right)+\frac{1}{2} \Delta z \cdot \mathcal{H}_{*} \cdot \Delta z+\ldots
$$

where $\Delta z \equiv\left(q-q_{*}, p-p_{*}\right)$.

We now consider the connection between the quadratic hamiltonian $h(q, p)$ of (13) and the local $2 \times 2$ dispersion matrix assumed in [4]. There, we asserted that in a conversion 
region a congruence transformation could be used to bring the dispersion matrix $D$ into the form

$$
\left(\begin{array}{ccc}
D_{\alpha}(\mathbf{z}) & \bar{\eta} & 0 \\
\bar{\eta} * & D_{\lambda}(\mathbf{z}) & 0 \\
0 & 0 & D_{\gamma}
\end{array}\right),
$$

with $D_{\alpha}, D_{\lambda}$ and $D_{\gamma}$ identified as the 'uncoupled' dispersion functions and $\tilde{\eta}$ the coupling constant. Focusing attention on the upper left block, the local $2 \times 2$ form is then expanded about the conversion point (defined in [4] as the point where the uncoupled rays cross). We identify this crossing point as the saddle point $Z_{*}$. The linearization (in the four-dimensional phase space $\mathbf{z}$ ) about $\mathbf{Z}_{*}$ gives (using $D_{\alpha}\left(\mathbf{Z}_{*}\right)=D_{\lambda}\left(\mathbf{Z}_{*}\right)=0$ ):

$$
\left(\begin{array}{cc}
\left(\mathbf{z}-\mathbf{Z}_{*}\right) \cdot \nabla_{z} D_{\alpha} & \bar{\eta} \\
\bar{\eta} * & \left(\mathrm{z}-\mathbf{Z}_{*}\right) \cdot \nabla_{z} D_{\lambda}
\end{array}\right) .
$$

We now assume that the variation of the linear functions $\left(\mathbf{z}-\mathbf{Z}_{*}\right) \cdot \nabla_{z} D_{\alpha}$ and $\left(\mathbf{z}-\mathbf{Z}_{*}\right) \cdot \nabla_{z} D_{\lambda}$ is predominantly in the osculating plane of the ray, implying that $\left(\mathbf{z}-\mathbf{Z}_{*}\right) \cdot \nabla_{\mathbf{z}} D_{\alpha} \approx(\mathrm{Z}(q, p)-$ $\left.\mathrm{Z}_{*}\right) \cdot \nabla_{z} D_{\alpha}$, etc. Hence, we replace the four-dimensional quantities in (15) with the local two-dimensional forms: $D_{\alpha}(q, p)=\Delta z \cdot \alpha=\left(q-q_{*}\right) \alpha_{q}+\left(p-p_{*}\right) \alpha_{p}$ and $D_{\lambda}(q, p)=\Delta z \cdot \lambda=$ $\left(q-q_{*}\right) \lambda_{q}+\left(p-p_{*}\right) \lambda_{p}$. The hamiltonian $h(q, p)$ is now identified as the determinant of the $2 \times 2$ dispersion matrix:

$$
h(q, p) \approx D_{\alpha}(q, p) D_{\lambda}(q, p)-|\tilde{\eta}|^{2}
$$

Using $D_{\alpha}\left(\mathrm{Z}_{*}\right)=D_{\lambda}\left(\mathrm{Z}_{*}\right)=0$, we find that

$$
h\left(q_{*}, p_{*}\right)=-|\bar{\eta}|^{2}
$$

and a little algebra shows that $(\Delta z \cdot \alpha)(\Delta z \cdot \lambda)$ can be written as $\Delta z \cdot \mathcal{H}_{*} \cdot \Delta z$ with

$$
\mathcal{H}_{*}=\left(\begin{array}{cc}
\alpha_{q} \lambda_{q} & \frac{1}{2}\left(\alpha_{q} \lambda_{p}+\alpha_{p} \lambda_{q}\right) \\
\frac{1}{2}\left(\alpha_{q} \lambda_{p}+\alpha_{p} \lambda_{q}\right) & \alpha_{p} \lambda_{p}
\end{array}\right) .
$$

Note the important result that: 


$$
\operatorname{det}\left(\mathcal{H}_{*}\right)=-\frac{1}{4}\left(\alpha_{q} \lambda_{p}-\alpha_{p} \lambda_{q}\right)^{2}=-\frac{1}{4}\left\{D_{\alpha}, D_{\lambda}\right\}^{2}
$$

This last quantity is the square of the Poisson bracket of $D_{\alpha}$ and $D_{\lambda}$, a quantity denoted $\mathcal{B}$ in [4]. Therefore, the normalized coupling is

$$
|\eta|^{2} \equiv \frac{|\bar{\eta}|^{2}}{|\mathcal{B}|}=-\frac{1}{2} \frac{h_{*}}{\left(-\operatorname{det} \mathcal{H}_{*}\right)^{1 / 2}}
$$

From this, we compute the transmission and conversion coefficients [4] (the reader should also refer to Fig. (1) for a graphical representation of the relationship between the incoming and outgoing wavefields):

$$
\psi_{\alpha}^{\text {out }}=\tau \psi_{\alpha}^{\text {in }}, \quad \psi_{\lambda}^{\text {out }}=\beta \psi_{\alpha}^{\text {in }}
$$

Here, $\psi_{\alpha}^{\text {in }}$ is the amplitude of the incoming field, meaning that the incoming electric field for polarization $\alpha$ is of the form $\mathrm{E}_{\alpha}^{i n}=\hat{\mathrm{e}}_{\alpha} \psi_{\alpha}^{i n}$, etc. The transmission and conversion coefficients are:

$$
\tau=e^{-\pi|\eta|^{2}}, \quad \beta(\eta) \equiv \frac{(2 \pi \tau)^{1 / 2}}{\eta \Gamma\left(-i|\eta|^{2}\right)} .
$$

In the expression for the conversion coefficient, $\beta$, we are lacking the phase of $\eta$. This will be discussed in a later paper.

For weak coupling, one can locate the saddle point and evaluate the magnitude of the coupling, using only information computed while following the incoming ray. Therefore, in this case there is no need to search for the saddle point. Taylor expanding $h(q, p)$ to $2^{\text {nd }}$ order about the origin (recall that the point $\mathrm{z}_{0}$ lies on a ray, hence $D\left(\sigma_{0}\right)=0$; and denote $\partial h / \partial q\left(\sigma_{0}\right) \equiv \partial h_{0} / \partial q$ etc. $):$

$$
h(q, p)=q \frac{\partial h_{0}}{\partial q}+p \frac{\partial h_{0}}{\partial p}+\frac{1}{2}\left(q^{2} \frac{\partial^{2} h_{0}}{\partial q^{2}}+2 q p \frac{\partial^{2} h_{0}}{\partial q \partial p}+p^{2} \frac{\partial^{2} h_{0}}{\partial p^{2}}\right)+\ldots
$$

We note that the quadratic terms involve the hessian evaluated at $\mathbf{z}_{0}$, which we denote $\mathcal{H}_{0}$. We first locate the saddle point $\left(q_{*}, p_{*}\right)$ by one-step Newton iteration:

$$
\left(\begin{array}{c}
q_{*} \\
p_{*}
\end{array}\right) \approx-\mathcal{H}_{0}^{-1} \cdot \nabla h_{0}
$$


and then note that

$$
h\left(q_{*}, p_{*}\right)=-\frac{1}{2} \nabla h_{0} \cdot \mathcal{H}_{0}^{-1} \cdot \nabla h_{0}
$$

For weak coupling the saddle is close to $\mathrm{z}_{0}$, therefore $\mathcal{H}_{0} \approx \mathcal{H}_{*}$ (correct to $\mathcal{O}\left(\Delta z^{2}\right)$ ). Therefore, we can evaluate the normalized coupling as

$$
|\eta|^{2}=\frac{1}{2} \frac{\left|\nabla h_{0} \cdot \mathcal{H}_{0}^{-1} \cdot \nabla h_{0}\right|}{\left|\operatorname{det} \mathcal{H}_{0}\right|^{1 / 2}}
$$

We note that all quantities in the above equation are evaluated on the incoming ray.

It remains to launch the transmitted ray. We can fix the initial conditions by searching along a straight line in phase space which connects the point $\mathrm{z}_{0}$ on the incoming ray with the saddle point $\mathbf{Z}_{*} \equiv \mathbf{Z}\left(q_{*}, p_{*}\right)=\mathbf{z}_{0}+q_{*} \hat{\mathrm{e}}_{q}+p_{*} \hat{\mathrm{e}}_{p}$. If there is a conversion occurring, this line will also puncture the other dispersion manifold (see Fig. (1)). Parameterize this connecting line as

$$
\mathrm{z}(s) \equiv \mathrm{z}_{0}+\left(\mathrm{Z}_{*}-\mathrm{z}_{0}\right) s, \quad-\infty<s<\infty
$$

The parameter value $s=0$ corresponds to $\mathrm{z}_{0}$, the parameter value $s=1$ is the saddle point $\mathbf{Z}_{*}$, hence the transmitted ray should lie in the vicinity of $s=2$. The initial conditions for the transmitted ray are fixed by finding the local zero of $D(\mathbf{z}(s))$ restricted to this line,

$$
f(s) \equiv D\left(\mathbf{z}_{0}+\left(\mathbf{Z}_{*}-\mathbf{z}_{0}\right) s\right)
$$

This line punctures the second dispersion manifold when $f=0$, which defines the parameter value $s=s^{\prime}$, and thereby fixes the initial condition of the transmitted ray $\mathbf{z}\left(s^{\prime}\right)=\mathbf{z}_{0}+\left(\mathbf{Z}_{*}-\right.$ $\left.z_{0}\right) s^{\prime}$. This is the initial condition for the transmitted ray. The transmitted ray is assigned an amplitude equal to that of the incoming ray at the conversion point $\mathrm{z}_{0}$ multiplied by the transmission coefficient $e^{-\pi|\eta|^{2}}$.

We now discuss the application of the algorithm to the MSW-IHW conversion in toroidal geometry, by first introducing a conceptual model to illustrate application of the algorithm, and then end with a very brief discussion of a more realistic toroidal model. 
Our simplified model is a variation of that used in [13], and is a two-dimensional variation of the model considered in [9]. Consider the two coupled wave equations ( $\bar{\eta}$ is constant in this model):

$$
\left(\begin{array}{cc}
D_{M}(\mathrm{x}, \mathrm{k}) & \bar{\eta} \\
\bar{\eta} & D_{H}(\mathrm{x}, \mathrm{k})
\end{array}\right)\left(\begin{array}{l}
\psi_{M} \\
\psi_{H}
\end{array}\right)=0,
$$

here $D_{M}$ is a simplified model of a magnetosonic (fast) wave confined to the poloidal plane of a torus with circular cross-section. In non-dimensional variables, we put $D_{M}(\mathrm{x}, \mathrm{k}) \equiv$ $1-\left(k_{x}^{2}+k_{y}^{2}\right)-\left(x^{2}+y^{2}\right)$. The $(x, y)$-dependence of $D_{M}$ provides for radial confinement of the MS waves. The second dispersion function $D_{H}$ is a simplified model of the IH resonance: $D_{H} \equiv x-x_{H}$. The ray hamiltonian is $D(\mathrm{x}, \mathbf{k})=D_{M} D_{H}-\tilde{\eta}^{2}$, giving the ray equations (see Eqs. (3))

$$
\begin{array}{ll}
\dot{x}=2\left(x-x_{H}\right) k_{x}, & \dot{y}=2\left(x-x_{H}\right) k_{y}, \\
\dot{k}_{x}=\left(1-k^{2}-r^{2}\right)-2\left(x-x_{H}\right) x, & \dot{k}_{y}=2\left(x-x_{H}\right) y .
\end{array}
$$

We note that in the vicinity of the IH resonance $x \approx x_{H}$, the evolution in the $k_{\mathrm{x}}$-direction dominates.

Ye and Kaufman first pointed out that resonance crossing could be treated as a two-step process in the ray phase space [14], involving a sequence of two conversions (see also [9]). In Fig. (2) a plot is shown of a ray undergoing such a pair of conversions. (The first conversion is shown in some detail, while the second is not, for clarity.) The incoming MS ray is launched (1) near the wall of the torus (numbers refer to locations in the figure). The ray is propagated using Eqs. (30). In the vicinity (2) of the first conversion, the trace of the dispersion matrix $\left(|\operatorname{Tr} \mathrm{D}|=\left|D_{M}+D_{H}\right|\right)$ exhibits a local minimum and fixes the location of the conversion point $z_{0}$. The osculating plane at $z_{0}$ is defined by $\dot{z}_{0}$ and $\ddot{z}_{0}$. A thumbnail contour plot of $h(q, p)$ in the osculating plane is shown, with proper orientation in the phase space. The contour is also shown in more detail as the inset at the upper right. This contour plot clearly displays a saddle structure. Note that the incoming ray starts in the upper left quadrant and exits as the converted ray in the upper right quadrant. The point of conversion 
is denoted with a large filled dot. The saddle point is indicated with a cross, and the initial conditions of the transmitted ray are indicated by an open dot. While the transmitted MS ray is launched in phase space, we show only its projection onto the $(x, y)$-plane at $(3)$ for clarity.

After the first conversion, the converted ray propagates predominantly in the $k_{x}$-direction (4). It has become an IH ray (a 'slow' wave). This IH ray undergoes a second conversion at (5) where it converts to the 'reflected' MS ray (6). The transmitted IH ray at (7) continues to propagate to large $k_{x}$ until it is eventually absorbed via Landau damping (assuming it is absorbed before undergoing further conversion of some kind). This propagation to regions of large $k_{x}$ implies that the wave fields develop fine scale structure near the conversion layer, an effect which makes direct numerical solution of the full wave equations difficult. The MS ray (8) subsequently undergoes further reflection at the outer wall of the tokamak and will re-enter the resonance layer.

We end with a very brief discussion of the application of the algorithm to a more realistic toroidal model. This model uses a $3 \times 3$ cold plasma dispersion matrix including finite $k_{\|}$. The ray equations are developed using magnetic coordinates, which allows for treatment of noncircular poloidal cross-sections and non-zero plasma current. A DH plasma is assumed and a Solovev equilibrium used for the plasma background. The algorithm has been implemented as a MATLAB module. This allows interactive display, for example, of the ray trajectories, the conversion monitor $F(\sigma)$, and the transmission coefficient $\tau$. Typical results are shown in Figs. (3) and (4).

To summarize, in this Letter we have proposed a ray-based algorithm for treating linear conversion in multi-dimensions. The algorithm assumes no prior knowledge of the location or geometry of the conversion region, and uses only information which is available by following the incoming ray. The algorithm assumes that, in the vicinity of the conversion, the ray is confined to a local two-dimensional plane in the phase space. The algorithm detects the presence of a conversion region, provides an evaluation of the connection coefficients, and fixes the initial conditions of the outgoing transmitted ray. Further development of 
the algorithm will include the amplitude propagation, the phase of the coupling coefficient, (which is needed for the conversion coefficient $\beta$ ), inclusion of thermal effects, and calculation of the wave fields in the immediate vicinity of conversion.

\section{ACKNOWLEDGMENTS}

The authors wish to thank Robert Littlejohn and Alan Weinstein for helpful conversations and suggestions. 


\section{REFERENCES}

[1] A. N. Kaufman, in Nonlinear and chaotic phenomena in plasmas, solids and fluids, W. Rozmus and J. A. Tuszynski eds. (World Scientific, 1991).

[2] R. G. Littlejohn, Phys. Rep. 138 (1986) 193.

[3] L. Friedland, Phys. Fluids 28 (1985) 3260.

[4] E. R. Tracy and A. N. Kaufman, Phys. Rev. E 48 (1993) 2196.

[5] A. Jaun, T. Hellsten, S. C. Chiu, Nuc. Fusion 38 (1998) 153.

[6] A. Jaun, T. Hellsten, S. C. Chiu, in "12th Topical Conference on RF Power in Plasmas", AIP Conf. Proc. 403 (1997) 281-284.

[7] P.T.Bonoli, M.Brambilla, E.Nelson-Melby et al. Phys. Plasmas 7 (2000) 1886.

[8] E.F.Jaeger, L.A.Berry, E.D'Azevedo,D.B.Batchelor, M.D.Carter, Phys. Plasmas 8 (2001) 1573.

[9] A. N. Kaufman, E. R. Tracy, J. J. Morehead and A. J. Brizard, Phys. Lett. A 252 (1999) 43.

[10] A. K. Ram, A. Bers, S. D. Schultz and V. Fuchs, Phys. Plasmas 3 (1996) 1976.

[11] Conversion can also occur when a single dispersion function has multiple branches which become nearly degenerate in some localized region (e.g. $D_{j}(\mathbf{z})=D_{j 1}(\mathbf{z}) D_{j 2}(\mathbf{z})$ with $D_{j 1}(\mathbf{z}) \simeq D_{j 2}(\mathbf{z}) \simeq 0$ locally). The algorithm described in this letter must be modified to deal with this type of conversion.

[12] B. O'Neill, Elementary differential geometry (Academic Press, New York, 1966).

[13] E. R. Tracy and A. N. Kaufman, in "13th Topical Conference on RF Power in Plasmas", AIP Conf. Proc. 485 (1999) 321-324.

[14] H. Ye and A. N. Kaufman, Phys. Rev. Lett. 60 (1988) 1642. 


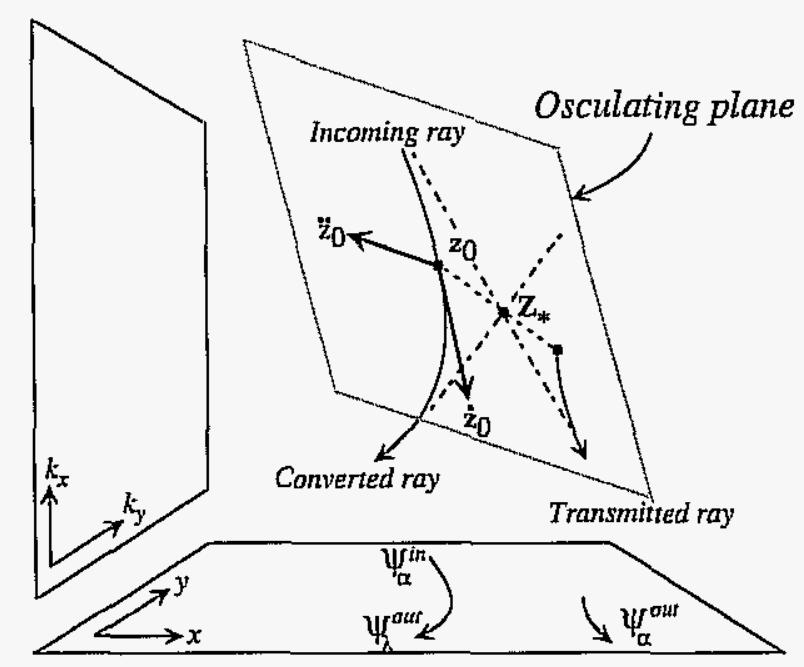

FIG. 1. Phase space diagram of the conversion region. The incoming ray enters from the upper part of the figure. The conversion monitor has identified the point $z_{0}$ as a candidate conversion point. At $z_{0}$, the two-dimensional osculating plane of the ray is defined by $\dot{z}_{0}$ and $\ddot{z}_{0}$. The local hamiltonian $h(q, p)$ is found to have a saddle structure (with asymptotes indicated by dot-dashed lines). The saddle point is at $\mathrm{Z}_{*}$. The transmitted ray is found by searching for a second zero of $h(q, p)$ along the line connecting $\mathbf{z}_{0}$ and $\mathbf{Z}_{*}$ (shown as a dashed line). A zero of $h(q, p)$ will also be a zero of the determinant $D(z)$ and, hence, a valid initial condition for the transmitted ray. See text for details.

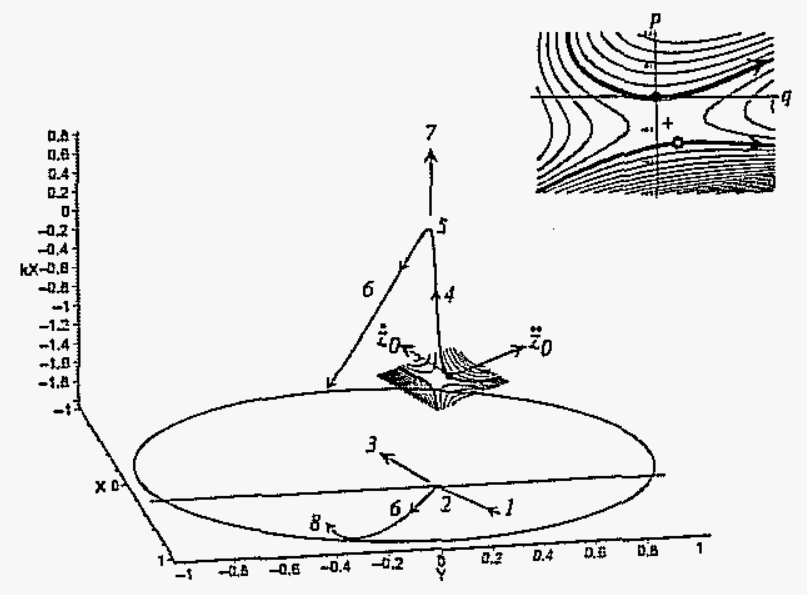

FIG. 2. MSW-IHW conversion in multi-dimensions. A phase space plot $\left(x, y, k_{x}\right)$ of the ray trajectory is shown. Also shown is the projection of the ray onto the $(x, y)$-plane. This figure was generated by numerically integrating the ray equations (30) using MAPLE. 


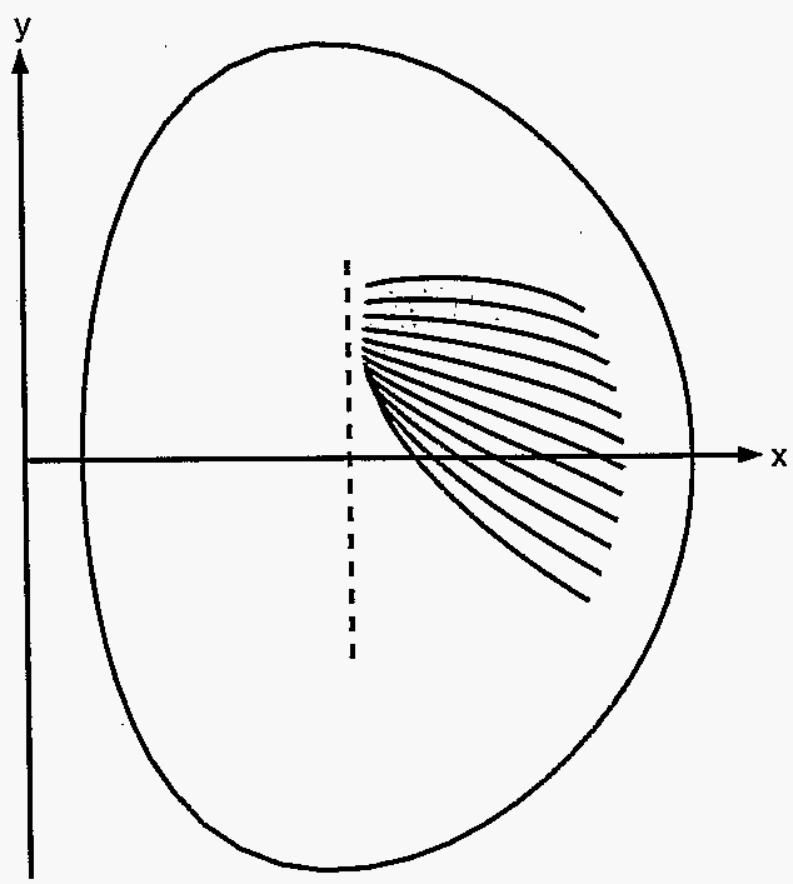

FIG. 3. Toroidal cross section and a dozen rays launched near the outer wall. The dashed line indicates roughly the location of the IH resonance layer. For clarity, the rays are followed only through the first conversion (i.e. the calculation is stopped when $k_{x}$ passes through zero). 


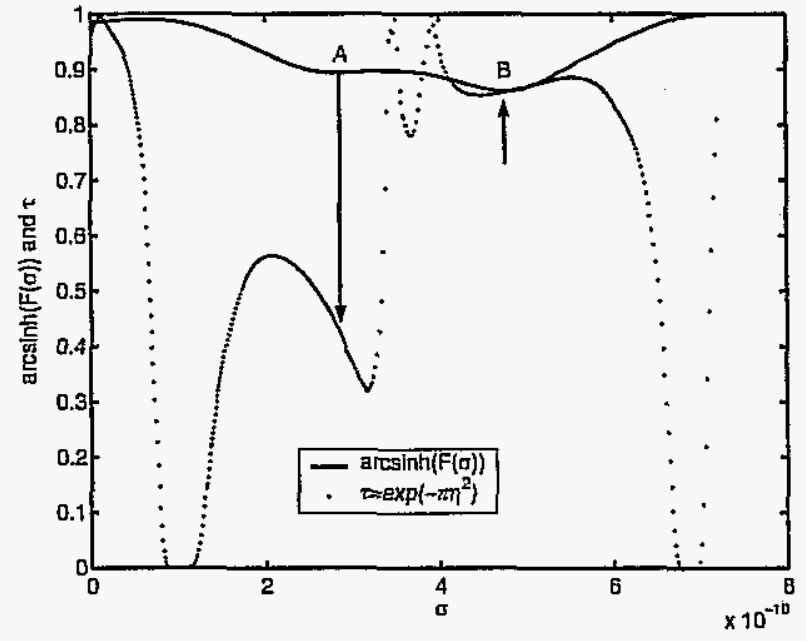

FIG. 4. Two time series (in ray parameter $\sigma$ ) showing the histories of the conversion monitor $|F(\sigma)|$ (solid line, arbitrary units), and the transmission coefficient $\tau$ (data points). The uppermost ray in Fig. (3) was used to generate the time series shown here by following it fully through the pair of conversions. The transmission coefficient is evaluated using Eqs. (26) and (22). Notice the two shallow minima of $|F(\sigma)|$ indicated on the figure at $A$ and $B$, with approximately $45 \%$ and $85 \%$ transmission, respectively. Both of these minima occur in the immediate vicinity of the resonance layer indicated by the dashed line in Fig. (3). 